\title{
Geomagnetic survey and geomagnetic model research in China
}

\author{
Zuowen $\mathrm{Gu}^{1}$, Zhijia Zhan ${ }^{1}$, Jintian $\mathrm{Gao}^{1}$, Wei $\mathrm{Han}^{1}$, Zhenchang An ${ }^{1,2}$, Tongqi Yao ${ }^{1}$, and Bin Chen ${ }^{1}$ \\ ${ }^{1}$ Institute of Geophysics, China Earthquake Administration, Beijing 100081, China \\ ${ }^{2}$ Institute of Geology and Geophysics, Chinese Academy of Sciences, Beijing 100029, China
}

(Received February 21, 2005; Revised September 23, 2005; Accepted October 11, 2005; Online published June 2, 2006)

\begin{abstract}
The geomagnetic survey at 135 stations in China were carried out in 2003 . These stations are with better environmental condition and small magnetic field gradient $(<5 \mathrm{nT} / \mathrm{m})$. In the field survey, the geomagnetic declination $D$, the inclination $I$ and the total intensity $F$ were measured. Ashtech ProMark2 differential GPS (Global Positioning System) was used in measuring the azimuth, the longitude, the latitude and the elevation at these stations. The accuracy of the azimuth is $0.1^{\prime}$. The geomagnetic survey data were reduced using the data at geomagnetic observatories in China. The mean standard deviations of the geomagnetic reduced values are: $<1.5 \mathrm{nT}$ for $F,<0.5^{\prime}$ for $D$ and $I$. Using the geomagnetic data which include the data at 135 stations and 35 observatories in China, and the data at 38 IGRF (International Geomagnetic Reference Field) calculation points in China's adjacent regions, the Taylor polynomial model and the spherical cap harmonic model were calculated for the geomagnetic field in China. The truncation order of the Taylor polynomial model is 5, and its original point is at $36.0^{\circ} \mathrm{N}$ and $104.5^{\circ} \mathrm{E}$. Based on the geomagnetic anomalous values and using the method of spherical cap harmonic $(\mathrm{SCH})$ analysis, the SCH model of the geomagnetic anomalous field was derived. In the SCH model, the pole of the spherical cap is at $36.0^{\circ} \mathrm{N}$ and $104.5^{\circ} \mathrm{E}$, and the half-angle is $30^{\circ}$, the truncation order $K=8$ is determined according to the mean square deviation between the model calculation value and the observation value, the AIC (Akaike Information Criterion) and the distribution of geomagnetic field.
\end{abstract}

Key words: Geomagnetic survey, geomagnetic model, Taylor polynomial model, spherical cap harmonic model, China.

\section{Introduction}

The geomagnetic survey and the geomagnetic field model are the foundation in geomagnetic research. The geomagnetic surveys were carried out in some regions of China since the beginning of the 20th century (Chen, 1944; Chen and Liu, 1948). The geomagnetic surveys in the whole China were carried out once every 10 years on the average during 1950 2000 (Tschu, 1979; An, 2001). In order to compile China geomagnetic chart for 2005.0, the geomagnetic surveys in China were carried out during 2002 2004. Modern equipments including GPS were used in geomagnetic survey. A lot of geomagnetic survey data with better accuracy and stability were obtained.

Geomagnetic field model is one of the important subjects in the study of geomagnetism (Langel, 1987). Geomagnetic field model is divided into the global model and the regional one. Beginning from 1968, the International Association of Geomagnetism and Aeronomy (IAGA) provides the global spherical harmonic models for each 5 years, i.e. the International Geomagnetic Reference Field (IGRF) and there is already the 9th generation IGRF (IAGA, 1996, 2000, 2003). In the research on regional geomagnetic field model, different scientists have used various mathematical methods and have obtained geomagnetic field models in various countries and regions (Alldredge, 1987; Barton, 1988; Haines,

Copyright (c) The Society of Geomagnetism and Earth, Planetary and Space Sciences (SGEPSS); The Seismological Society of Japan; The Volcanological Society of Japan; The Geodetic Society of Japan; The Japanese Society for Planetary Sciences; TERRAPUB.
1990; Haines and Newitt, 1986; Newitt et al., 1996; Kote and Haok, 2000). In the research on geomagnetic field model in China, Chen (1948) first established the Taylor polynomial model of geomagnetic field in Beipai region of Chongqing for 1946. Chinese scientists have studied the geomagnetic models by using the Taylor polynomial method, the rectangular harmonic analysis, the spline method and the spherical cap harmonic analysis (An et al., 1991; Xia et al., 1988; Xu et al., 2003; Gu et al., 2004; Gao et al., 2005). The above-mentioned results have greatly promoted the development of the research on geomagnetic field model.

The geomagnetic survey in China in 2003 was briefly described in the Section 2; the Section 3 gives the method and result of Taylor polynomial analysis of geomagnetic field in China; the model of geomagnetic anomalous field in China is calculated based on the spherical cap harmonic method in the Section 4; finally the discussion and the conclusions are also given respectively in the Sections 5 and 6.

\section{Geomagnetic Survey in China in $\mathbf{2 0 0 3}$}

In geomagnetic survey, G-856 magnetometer is used for measuring the total intensity $F$ of geomagnetic field, its resolution is $0.1 \mathrm{nT}$ and the accuracy is $0.5 \mathrm{nT}$. DI magnetometer is used for measuring the declination $D$ and inclination $I$, the resolution is $0.1^{\prime}$ and the accuracy is $0.2^{\prime}$. GPS is used for measuring the azimuth of the reference mark so as to determine the geomagnetic declination, as well as to measure the longitude, the latitude and the elevation at 


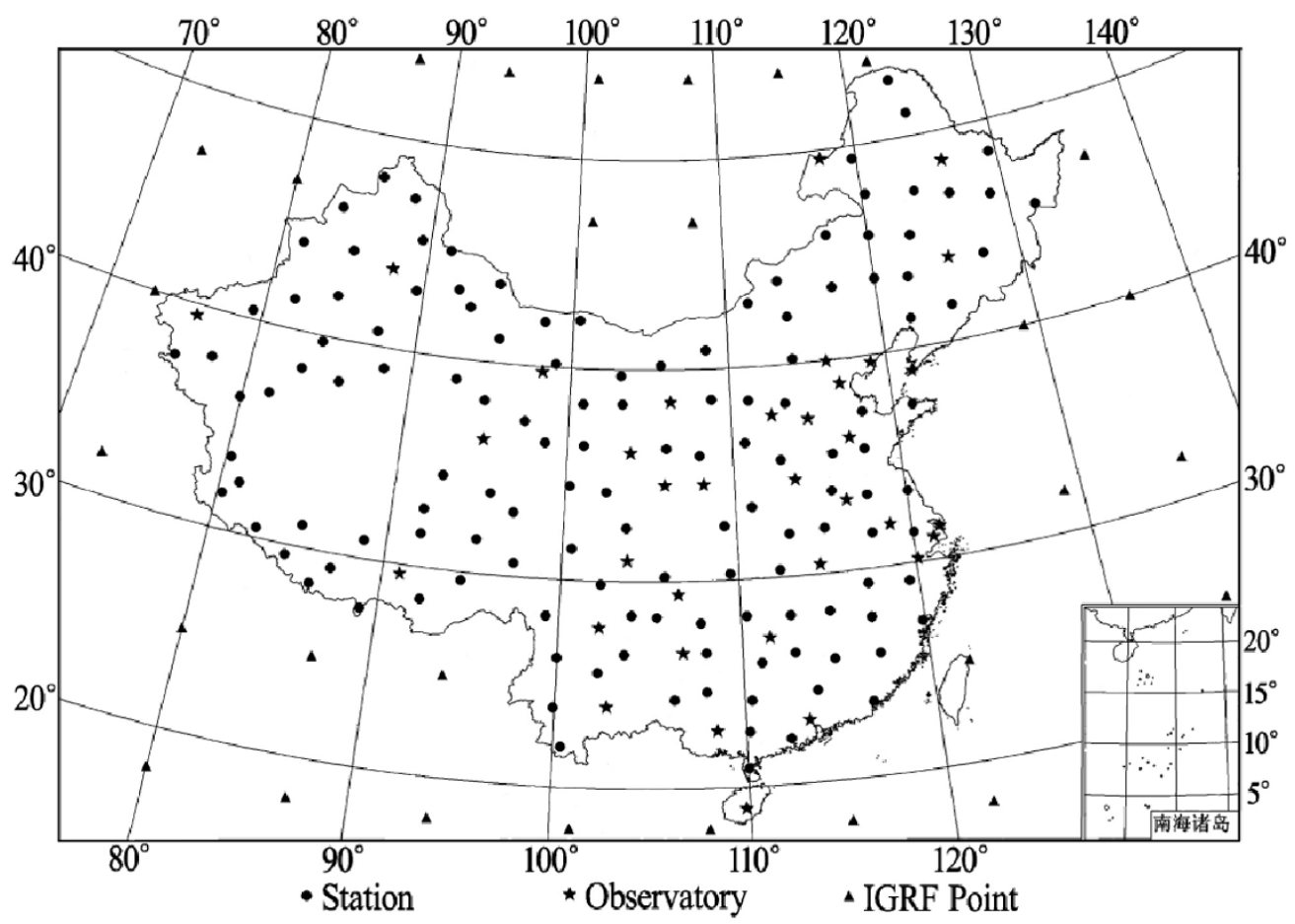

Fig. 1. Distribution of the geomagnetic stations and observatories in China.

the station (Newitt et al., 1996; Gu et al., 2006), its vertical locating accuracy is $10 \mathrm{~mm}+1 \mathrm{ppm}$ and the horizontal locating accuracy is $5 \mathrm{~mm}+1 \mathrm{ppm}$. The magnetometers are calibrated before and after the survey in order to determine the differences between these magnetometers and the standard one, and the differences were corrected in data processing.

In China, the 135 geomagnetic stations (Fig. 1) were established in 2003. Each station was carefully selected. The environmental condition around each station is good without any electromagnetic noise. The geomagnetic gradient around each station is $<5 \mathrm{nT} / \mathrm{m}$ : the range of the horizontal gradient is $0.4 \sim 4.9 \mathrm{nT} / \mathrm{m}$, and its average is $2.1 \mathrm{nT} / \mathrm{m}$; the range of vertical gradient is $0.2 \sim 4.9 \mathrm{nT} / \mathrm{m}$, and its average is $2.3 \mathrm{nT} / \mathrm{m}$. The distance between the station and the reference mark is $>200 \mathrm{~m}$.

The survey at each station includes geomagnetic threecomponent and GPS observations. The sensor position of G-856 magnetometer is ensured at the same position with the coil position of $D I$ magnetometer, the $D I$ magnetometer is ensured at the same position with the centering position of GPS.

G-856 magnetometer is used to measure $F$ simultaneously with $I . D I$ magnetometer is used to measure $D$ and $I$ with 8 group-data, including 4 group-data of positive and inverted telescope. Meanwhile, the azimuths of the reference mark are measured by GPS before and after measuring $D$ and $I$ respectively.

GPS is used twice to measure the azimuths of the reference mark at each station. The observation results of GPS show that the distances between the station and the reference mark are $>200 \mathrm{~m}$. The number of satellite received by GPS is $5 \sim 10$, the PDOP (Positioning Dilution of Precision) value is $1.7 \sim 5.4$. The data measured by GPS are processed by using a notebook computer in field. The results show that the differences between two azimuths measured by GPS at various sites are $0.0^{\prime \prime} \sim 5.9^{\prime \prime}$, the average is $1.6^{\prime \prime}$.

The geomagnetic data of field survey were reduced by using the data at the observatories in China. The mean standard deviations of geomagnetic reduced values are: $<1.5$ nT for $F ;<0.5^{\prime}$ for $D$ and $I$. This shows that the geomagnetic survey data are reliable and accurate.

\section{Taylor Polynomial Analysis of Geomagnetic Field in China \\ 3.1 Method}

Taylor polynomial model of geomagnetic field can be expressed as:

$$
F=\sum_{n=0}^{N} \sum_{m=0}^{n} A_{n m}\left(\varphi-\varphi_{0}\right)^{n-m}\left(\lambda-\lambda_{0}\right)^{m}
$$

where $A_{n m}$ is the Taylor polynomial coefficient, $N$ is the truncation order of the Taylor polynomial, $\varphi$ and $\lambda$ are respectively the longitude and the latitude at the station, $\varphi_{0}$ and $\lambda_{0}$ are respectively the longitude and the latitude at the original point. $F$ can represent any element of geomagnetic field.

\subsection{Result}

According to the geomagnetic data, which include the data at 135 stations and 35 observatories in China, and the data at 38 IGRF calculation points in China's adjacent regions, the Taylor polynomial model of the geomagnetic field in China for 2003 were calculated.

Table 1 shows the RMS (root mean square) $\sigma$ of the Taylor polynomial model for geomagnetic field in China with different order $K$. It is seen from Table 1 that when the order $K$ increases, all of $\sigma_{x}, \sigma_{y}$ and $\sigma_{z}$ decrease; when $K \geq 5$, 
(a)

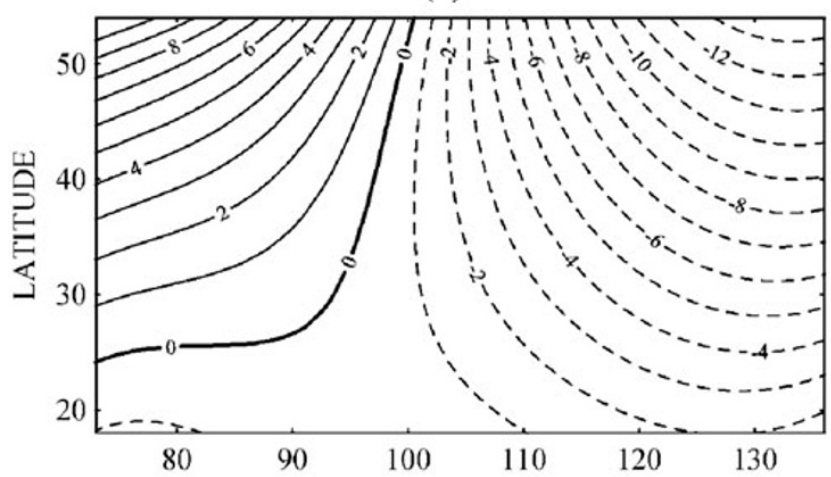

(c)

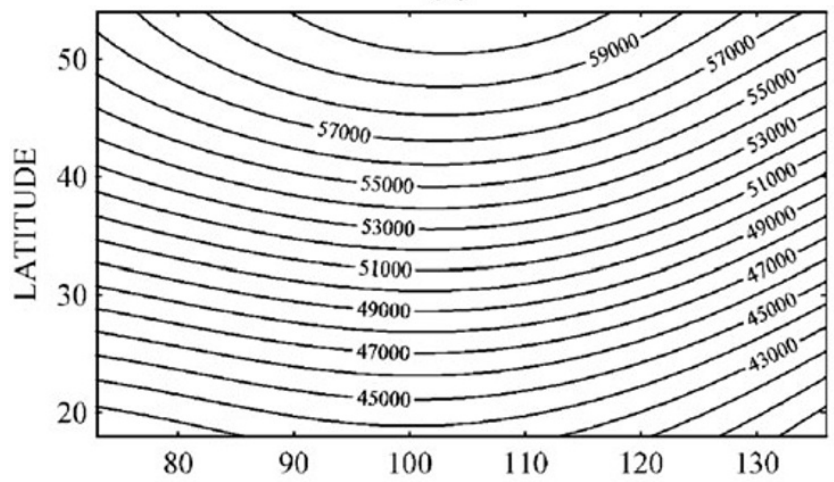

(e)

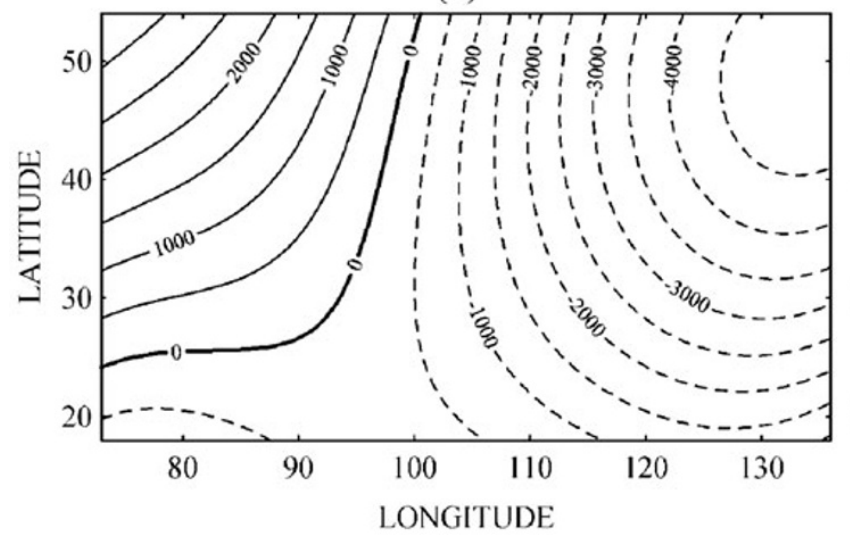

(b)

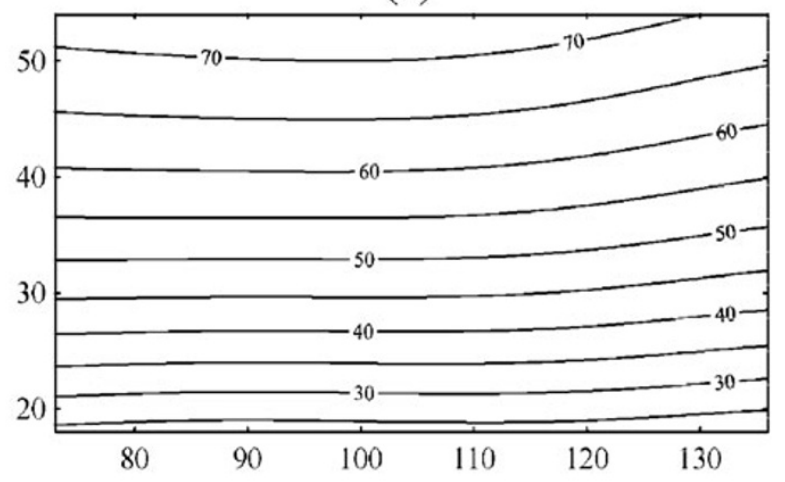

(d)

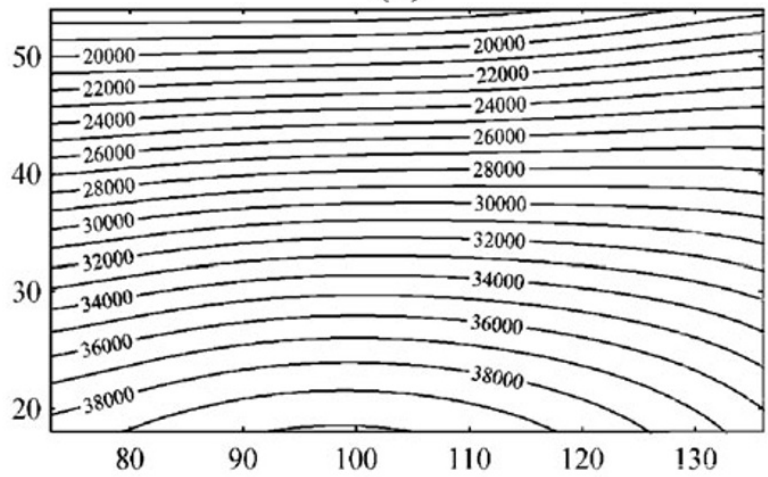

(f)

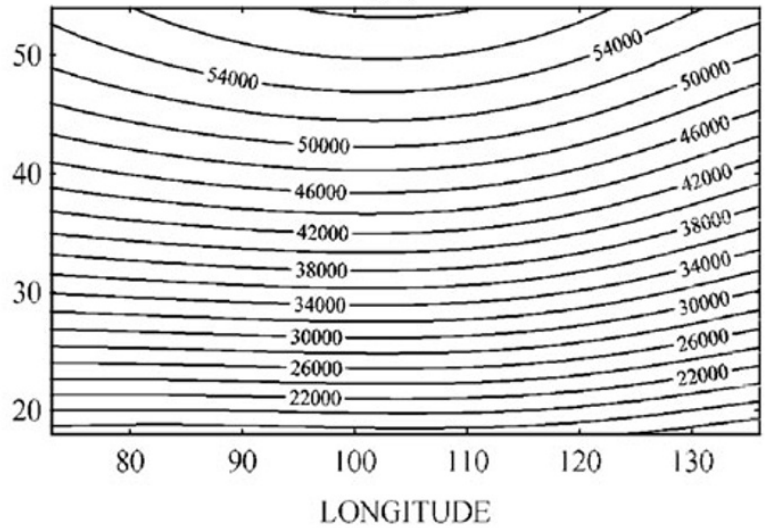

Fig. 2. Geomagnetic charts derived from the 5-order Taylor polynomial model in China. (a) $D, \Delta=1^{\circ}$; (b) $I, \Delta=5^{\circ}$; (c) $F, \Delta=1,000 \mathrm{nT}$; (d) $X$, $\Delta=1,000 \mathrm{nT}$; (e) $Y, \Delta=500 \mathrm{nT}$; (f) $Z, \Delta=1,000 \mathrm{nT}$.

$\sigma_{x}, \sigma_{y}$ and $\sigma_{z}$ are small and stable. The 5-order Taylor polynomial model is taken as the geomagnetic field model in China for 2003 (see Discussion). Table 2 shows the coefficients of this model. Figure 2 shows the geomagnetic charts derived from this model in China. The distribution characters of various geomagnetic components can be seen from Fig. 2 that: the declination $D$ and the east component $Y$ are basically distributed along the longitude; $D$ decreases from $8^{\circ}$ in the west to $-12^{\circ}$ in the east; $Y$ decreases from 2,000 $\mathrm{nT}$ in the west to $-4,000 \mathrm{nT}$ in the east. The geomagnetic total intensity $F$, the inclination $I$, the north component $X$ and the vertical component $Z$ are basically distributed along the latitude; $F$ increases from $43,000 \mathrm{nT}$ in the south to $59,000 \mathrm{nT}$ in the north; $X$ decreases from 38,000 nT in the south to $20,000 \mathrm{nT}$ in the north; $Z$ increases from 22,000
$\mathrm{nT}$ in the south to $54,000 \mathrm{nT}$ in the north; $I$ increases from $30^{\circ}$ in the south to $70^{\circ}$ in the north.

Figure 3 shows the distribution of the geomagnetic anomalous field in China, the values of this anomalous field are the differences between the observed values and those of the 5-order Taylor polynomial model of geomagnetic field in China (CGRF). In Fig. 3, there are either positive or negative geomagnetic anomalies in most regions of China, the distribution is reasonable.

\section{Spherical Cap Harmonic Analysis on the Geo- magnetic Field in China \\ 4.1 Method}

The geomagnetic anomalous field $(\Delta X, \Delta Y, \Delta Z)$ under the spherical cap coordinate system can be expressed as: 
Table 1. RMS $\sigma$ of Taylor polynomial model for geomagnetic field in China with different order $K$.

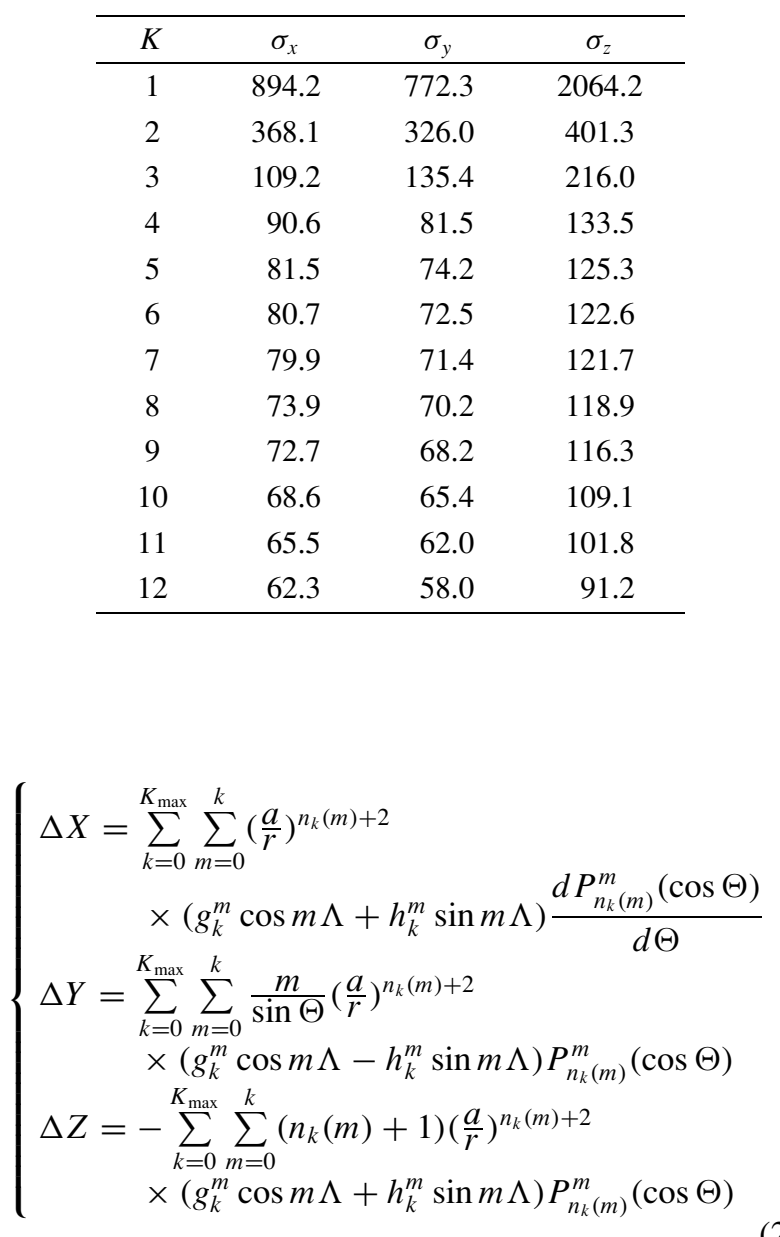

In formula (2), $\Lambda$ and $\Theta$ are respectively the longitude and the colatitude under the spherical cap coordinate system, $a$ is the reference radius of the Earth $(a=6,371.2$ $\mathrm{km}), r$ is the radial distance apart from the Earth's center; $P_{n_{k}(m)}^{m}(\cos \Theta)$ is the Legendre function of Schmidt association of non-integer order $n_{k}(m)$ and integer $m$. $K_{\max }$ represents the truncation order of the spherical cap harmonic analysis. The spherical cap harmonic model has $\left(K_{\max }+1\right)^{2}$ coefficients altogether. These coefficients $g_{k}^{m}$ and $h_{k}^{m}$ are determined by the least square method based on the observational values of geomagnetic field.

The spherical cap harmonic expressions of other components of geomagnetic anomalous field can be obtained by using the following formulas:

$$
\begin{gathered}
\Delta F=\frac{X}{F} \Delta X+\frac{Y}{F} \Delta Y+\frac{Z}{F} \Delta Z \\
\Delta D=\frac{X}{H^{2}} \Delta Y-\frac{Y}{H^{2}} \Delta X \\
\Delta I=\frac{H}{F^{2}} \Delta Z-\frac{X Z}{H F^{2}} \Delta X-\frac{Y Z}{H F^{2}} \Delta Y \\
\Delta H=\frac{X}{H} \Delta X+\frac{Y}{H} \Delta Y
\end{gathered}
$$

The $\Delta X, \Delta Y$ and $\Delta Z$ in the above-mentioned formulas are calculated by using formula (2), $X, Y, Z, H$ and $F$ are calculated by using the IGRF 2000 .
Table 2. The coefficients of the 5-order Taylor polynomial model in China

\begin{tabular}{lrrr}
\hline$A_{n m}$ & $X$ & $Y$ & $Z$ \\
\hline$A_{00}$ & 31048.7 & -1093.6 & 43236.2 \\
$A_{10}$ & -23501.7 & 5551.5 & -56284.2 \\
$A_{11}$ & 15815.3 & -13048.6 & -21361.7 \\
$A_{20}$ & 4634.2 & 13969.1 & -12187.0 \\
$A_{21}$ & 16312.7 & 621.6 & 40160.9 \\
$A_{22}$ & 70665.8 & 120977.5 & -19344.5 \\
$A_{30}$ & 6281.7 & -16216.6 & 16170.3 \\
$A_{31}$ & -38361.8 & -407.9 & 67924.1 \\
$A_{32}$ & 8427.1 & -15747.7 & -2417.8 \\
$A_{33}$ & -5937.9 & 17448.8 & 18405.9 \\
$A_{40}$ & -4541.3 & -5367.1 & 6968.6 \\
$A_{41}$ & 1416.7 & 9181.4 & 12985.7 \\
$A_{42}$ & 9150.2 & -14135.3 & -26479.2 \\
$A_{43}$ & -16701.0 & -18465.4 & 13101.8 \\
$A_{44}$ & 106.0 & -8109.9 & -2000.0 \\
$A_{50}$ & -3975.7 & -3820.1 & -18144.4 \\
$A_{51}$ & 27436.9 & 7576.6 & -23852.5 \\
$A_{52}$ & -23748.4 & 14220.5 & -1650.7 \\
$A_{53}$ & -168.6 & 10888.1 & 13023.1 \\
$A_{54}$ & -28063.2 & 5911.8 & 37837.7 \\
$A_{55}$ & -10620.1 & -11570.2 & 17957.0 \\
\hline & & &
\end{tabular}

Referring to the IGRF2000 and the spherical cap harmonic model $(A S C H)$ of geomagnetic anomalous field, the spherical cap harmonic model (SCHRF) can be obtained:

$$
S C H R F=I G R F 2000+A S C H
$$

\subsection{Result}

Using the above geomagnetic data, which include the data at 135 stations and 35 observatories in China, and the data at 38 IGRF calculation points in China's adjacent regions, the spherical cap harmonic model is calculated.

Table 3 shows the RMS $\sigma$ of spherical cap harmonic model for geomagnetic field. It is seen in Table 3 that the lager the order $K$ is, the smaller the $\sigma_{x}, \sigma_{y}$ and $\sigma_{z}$ are; when $K \geq 8$, the $\sigma_{x}, \sigma_{y}$ and $\sigma_{z}$ are small and stable. Figure 4 shows the geomagnetic field derived from the 8order SCH model in China (see Discussion). Comparing Fig. 4 with Fig. 2, the distribution of the same component of geomagnetic field in China in Fig. 4 is very similar with that in Fig. 2.

Table 4 shows the RMS $\sigma$ of spherical cap harmonic model for geomagnetic anomalous field. It is seen in Table 4 that the lager the order $K$ is, the smaller the $\sigma_{x}, \sigma_{y}$ and $\sigma_{z}$ are; when $K \geq 8$, the $\sigma_{x}, \sigma_{y}$ and $\sigma_{z}$ are small and stable. The 8-order spherical cap harmonic model is selected as the mathematical model for the geomagnetic anomalous field ( $\Delta X, \Delta Y, \Delta Z$ ) in China for 2003 (see Discussion). Table 5 shows the coefficients of this model. Based on the spherical cap harmonic model (SCHA) of geomagnetic anomalous field in China, the chart of geomagnetic anomalous field in China is drawn (Fig. 5). It is seen from Fig. 5 that the distribution characters of geomagnetic anomalous field 

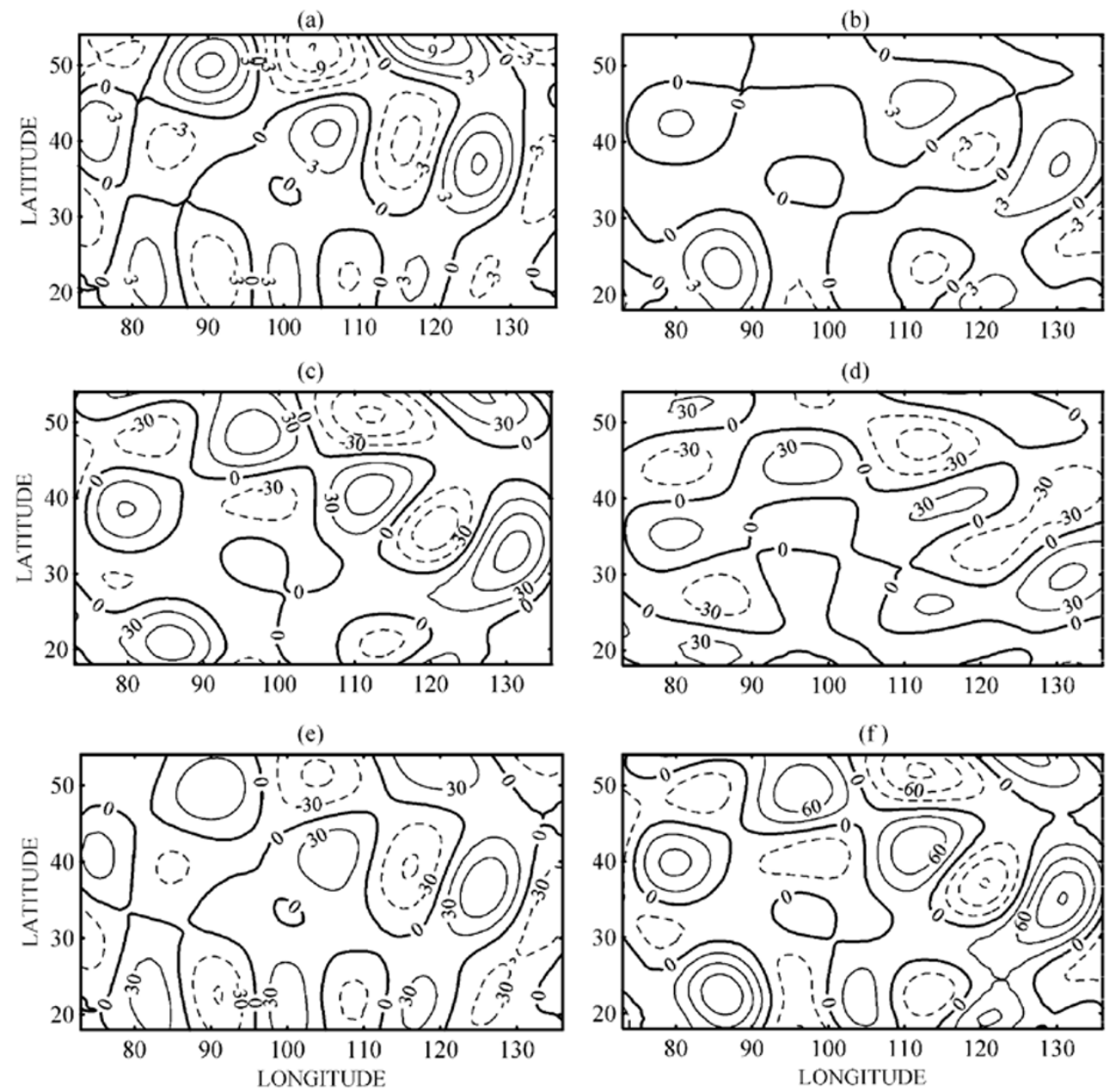

Fig. 3. Geomagnetic anomalous field based on the 5-order Taylor polynomial model as the normal reference field in China. (a) $\Delta D, \Delta=3^{\prime}$; (b) $\Delta I$, $\Delta=3^{\prime}$; (c) $\Delta F, \Delta=30 \mathrm{nT}$; (d) $\Delta X, \Delta=30 \mathrm{nT}$; (e) $\Delta Y, \Delta=30 \mathrm{nT}$; (f) $\Delta Z, \Delta=30 \mathrm{nT}$.

Table 3. RMS $\sigma$ of spherical cap harmonic model for geomagnetic field in China.

\begin{tabular}{cccc}
\hline$K$ & $\sigma_{x}$ & $\sigma_{y}$ & $\sigma_{z}$ \\
\hline 1 & 3931.4 & 1845.4 & 5997.7 \\
2 & 1782.7 & 1072.1 & 2582.6 \\
3 & 925.5 & 602.3 & 719.5 \\
4 & 360.8 & 245.0 & 472.8 \\
5 & 173.0 & 134.7 & 257.5 \\
6 & 124.2 & 99.1 & 154.8 \\
7 & 93.7 & 76.9 & 122.4 \\
8 & 90.4 & 75.2 & 115.7 \\
9 & 84.0 & 73.4 & 110.7 \\
10 & 78.6 & 72.6 & 100.8 \\
11 & 78.8 & 67.7 & 89.1 \\
12 & 77.8 & 64.7 & 82.8 \\
\hline
\end{tabular}

are negative anomalies in most of the areas for various components.

\section{Discussion}

In calculating the geomagnetic field model, the results appear larger misfit of the geomagnetic anomalies in the
Table 4. RMS $\sigma$ of spherical cap harmonic model for geomagnetic anomalous field in China.

\begin{tabular}{cccc}
\hline$K$ & $\sigma_{x}$ & $\sigma_{y}$ & $\sigma_{z}$ \\
\hline 1 & 89.1 & 80.5 & 136.2 \\
2 & 89.2 & 79.6 & 133.0 \\
3 & 86.9 & 79.4 & 130.8 \\
4 & 87.2 & 77.0 & 126.9 \\
5 & 86.0 & 77.3 & 125.4 \\
6 & 86.1 & 77.2 & 123.9 \\
7 & 85.2 & 76.6 & 121.2 \\
8 & 84.9 & 76.9 & 119.1 \\
9 & 85.0 & 76.7 & 115.1 \\
10 & 83.1 & 75.2 & 111.9 \\
11 & 80.7 & 75.1 & 110.1 \\
12 & 79.2 & 72.9 & 102.5 \\
\hline
\end{tabular}

boundary area by only using the geomagnetic data at 135 stations and 35 observatories in China. After adding the calculation values at some IGRF points in the boundary area, the above-mentioned misfit of the geomagnetic anomalies is reduced ( $\mathrm{Gu}$ et al., 2004; Gao et al., 2005). In order to search for the rationality of the number of the added IGRF 
Table 5. The coefficients of the 8-order spherical cap harmonic model of geomagnetic anomalous field in China.

\begin{tabular}{rrrr}
\hline$K$ & $M$ & $g_{k}^{m}$ & $h_{k}^{m}$ \\
\hline 0 & 0 & 358.5 & \\
1 & 0 & -406.8 & \\
1 & 1 & -104.7 & 63.2 \\
2 & 0 & 702.2 & \\
2 & 1 & 255.1 & -57.6 \\
2 & 2 & -58.5 & 14.6 \\
3 & 0 & -892.7 & \\
3 & 1 & -397.9 & 68.6 \\
3 & 2 & 160.8 & -37.4 \\
3 & 3 & -14.2 & -45.7 \\
4 & 0 & 874.0 & \\
4 & 1 & 414.9 & -61.9 \\
4 & 2 & -209.2 & 59.4 \\
4 & 3 & 56.0 & 82.9 \\
4 & 4 & -4.0 & 21.7 \\
5 & 0 & -595.4 & \\
5 & 1 & -318.2 & 50.8 \\
5 & 2 & 141.0 & -73.7 \\
5 & 3 & -83.8 & -86.7 \\
5 & 4 & 49.4 & -24.3 \\
5 & 5 & 17.8 & -12.1 \\
6 & 0 & 289.3 & \\
6 & 1 & 165.8 & -35.0 \\
\hline & & &
\end{tabular}

\begin{tabular}{rrrr}
\hline$K$ & $M$ & $g_{k}^{m}$ & $h_{k}^{m}$ \\
\hline 6 & 2 & -49.2 & 62.6 \\
6 & 3 & 77.7 & 48.2 \\
6 & 4 & -70.4 & 23.1 \\
6 & 5 & -23.2 & 27.0 \\
6 & 6 & 19.9 & -9.4 \\
7 & 0 & -82.3 & \\
7 & 1 & -61.8 & 16.8 \\
7 & 2 & -1.4 & -32.4 \\
7 & 3 & -43.0 & -12.8 \\
7 & 4 & 47.2 & -10.6 \\
7 & 5 & 17.1 & -27.3 \\
7 & 6 & -8.6 & 9.5 \\
7 & 7 & -0.3 & 5.8 \\
8 & 0 & 13.5 & \\
8 & 1 & 10.8 & -4.2 \\
8 & 2 & 4.1 & 9.8 \\
8 & 3 & 10.6 & -1.4 \\
8 & 4 & -14.0 & 2.0 \\
8 & 5 & -5.8 & 11.2 \\
8 & 6 & 1.5 & -6.8 \\
8 & 7 & -2.4 & 1.4 \\
8 & 8 & 3.4 & -3.1 \\
& & & \\
\hline & & &
\end{tabular}

points and the distribution, a model calculation is done for some groups of selecting 28 48 IGRF points and their homogeneous distribution. The model calculation results for different groups are analyzed and compared. The comprehensive analysis and comparison show that the effects become the best when adding 38 IGRF points as shown in Fig. 1. This shows that it is necessary and rational to add IGRF points appropriately.

Taylor polynomial model is a common one of geomagnetic field because it is convenient in calculation and application. In the research on geomagnetic field model in China, Chinese scientists have studied the Taylor polynomial models in China (Chen, 1948; Xia et al., 1988; Xu et al., 2003). In these studies of the Taylor polynomial model, the truncation order $K$ of the models was taken as $K=2$ (Chen, 1948), $K=3$ (Xia et al., 1988) and $K=4$ (Xu et $a l ., 2003)$. The truncation order of the Taylor polynomial model is taken as $K=5$ in this paper. The larger the $K$ is, the higher the space resolution is, and the smaller the mean square deviation between the model calculation value and the observation value is. Therefore, the 5-order Taylor polynomial model in this paper can better describe the geomagnetic field in China.

The spherical cap harmonic ( $\mathrm{SCH}$ ) analysis is an effective method in the research on regional geomagnetic field. The calculation results show that $\mathrm{SCH}$ method can not only describe the distribution of geomagnetic field in a large range (such as the whole China or Asia), but can also describe the distribution of geomagnetic field in a smaller range as Beijing-Tianjin-Hebei region (Gu et al., 2004).

In order to compare the distribution of the geomagnetic anomalous field in China derived from the Taylor polynomial model (Fig. 3) and the SCHA model (Fig. 5), we analyze the similarity and the difference between Figs. 3 and 5 . It is seen from Figs. 3 and 5 that the geomagnetic anomalous charts basically have the similar shape for the same component. In Fig. 3, there are either positive or negative geomagnetic anomalies in most regions of China, the distribution is reasonable. In Fig. 5, the geomagnetic anomalous fields (such as $\Delta F, \Delta Y$ and $\Delta Z$ etc.) in most regions of China are negative, the distribution of geomagnetic anomaly is not equivalent. It shows that there is difference between the anomalous fields in Figs. 3 and 5. In fact, Fig. 5 is based on IGRF as the normal reference field while Fig. 3 is based on CGRF as the normal reference field. Taking IGRF as the normal reference field, it can study the anomalous field with a large scale; while taking CGRF as the normal reference field, it can explore the anomalous field with a smaller scale.

One of the key problems in establishing the geomagnetic field model is to determine the truncation order $K$. The larger the $K$ is, the higher the space resolution is, and the smaller the mean square deviation between the model calculation value and the observation value is. However, when $K$ is large, the model calculation results are relatively unstable. In order to reasonably determine $K, K$ was respectively taken as $1 \sim 12$ for the spherical cap harmonic model in model calculation. Figure 6(a) shows the decrease of $\sigma$ (RMS values) of the SCH model of the geomagnetic field with different truncation order $K$. It is seen from Fig. 6(a) 
(a)

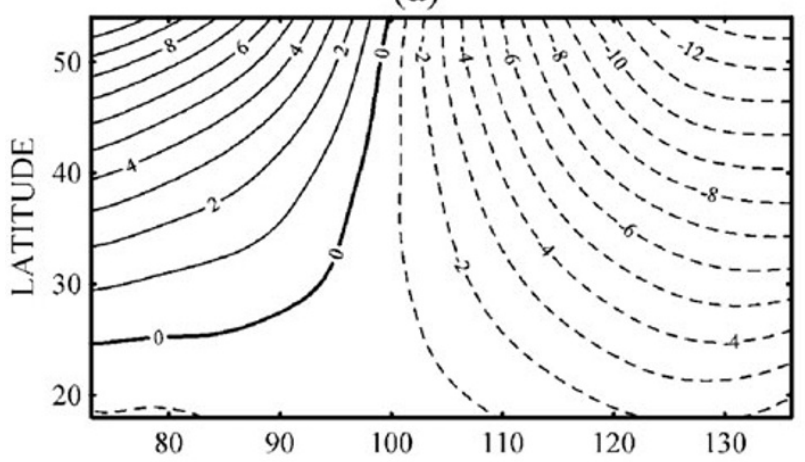

(c)

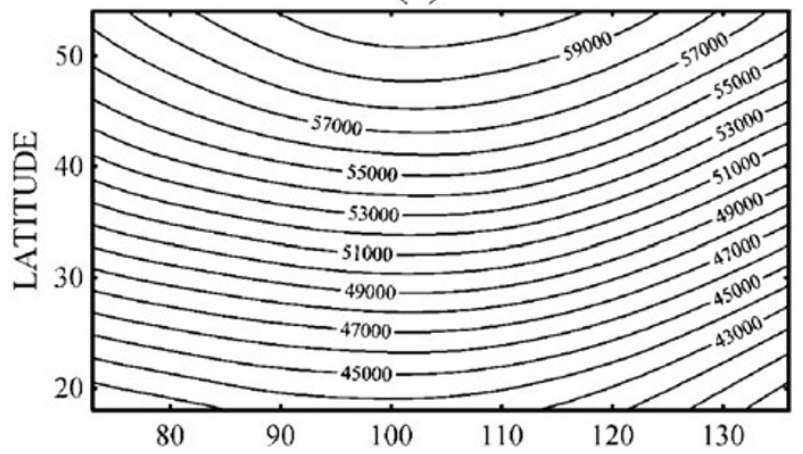

(e)

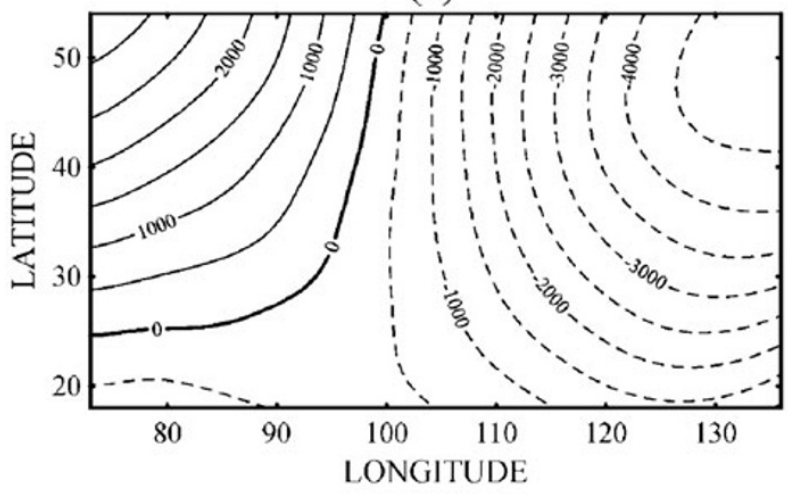

(b)

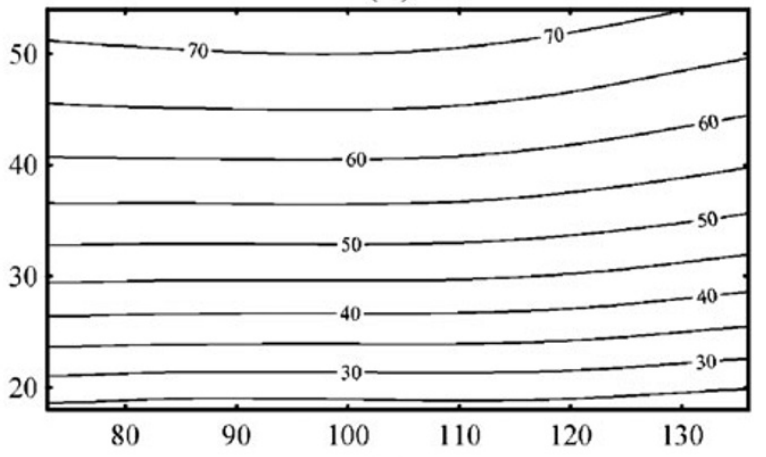

(d)

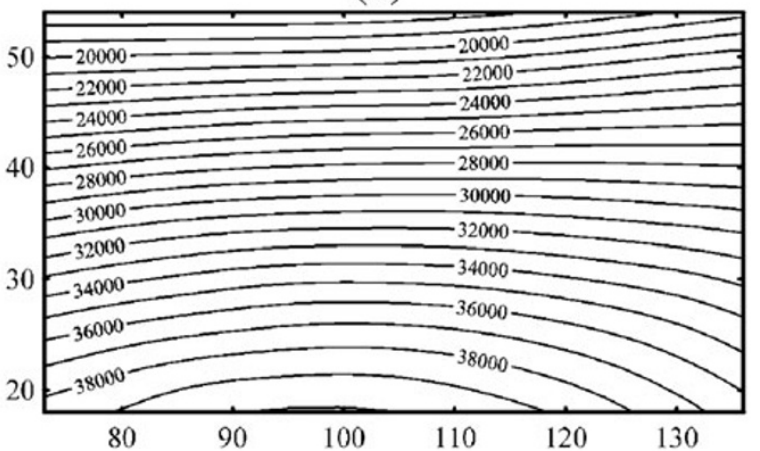

(f)

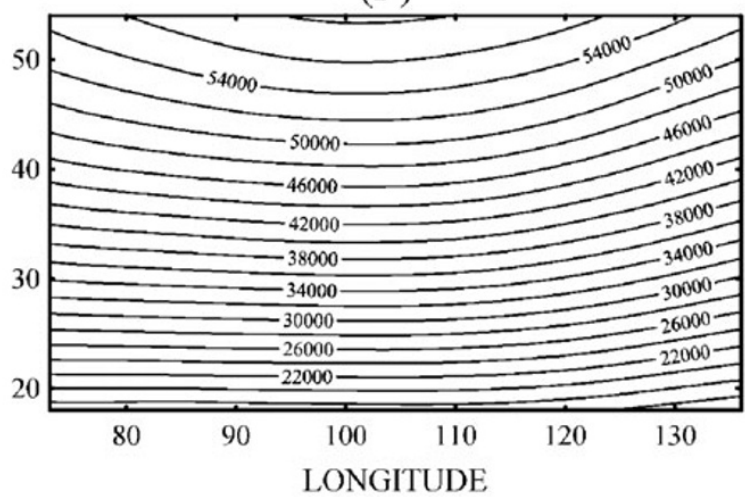

Fig. 4. Geomagnetic field derived from the 8 -order SCH model in China. (a) $D, \Delta=1^{\circ}$; (b) $I, \Delta=5^{\circ}$; (c) $F, \Delta=1,000 \mathrm{nT}$; (d) $X, \Delta=1,000 \mathrm{nT}$; (e) $Y, \Delta=500 \mathrm{nT}$; (f) $Z, \Delta=1,000 \mathrm{nT}$.

that when $K \geq 8$, the $\sigma_{x}, \sigma_{y}$ and $\sigma_{z}$ are small and stable. In order to determine the truncation order $K$, we also calculate AIC (Akaike Information Criteria) (Akaike, 1974; Miura et al., 2000) for different truncation order $K$ taken as 1-12 respectively. The AIC for each $K$ is shown in Table 6 and Fig. 6(b). Figure 6(b) shows that the AIC decreases when $K$ increases; when $K \geq 7$, the AIC is nearly stable. Meanwhile, we compare the distribution of the geomagnetic field and the anomalous field derived from the SCH models with $K \geq 8$. As an example, Figs. 3(a) and 7 give the comparison of the declination distribution of the $\mathrm{SCH}$ anomalous field models for $K=8$ (Fig. 3(a)), $K=9$ and 10 (Fig. 7). The comparison results show that the distribution with $K=8$ is reasonable. However, the distribution with $K \geq 9$ appears some misfit, the larger the $K$ is, the larger the misfit is. Therefore, $K=8$ is finally taken as the truncation order of the SCH model. It is seen from Table 7 that the RMS values of the 8-order SCHN model ( $\mathrm{SCH}$ model
Table 7. Comparison of $\sigma$ (RMS) for various models in China for 2003. The 8-order SCHN is the 8-order SCH model of geomagnetic field, the 8 -order SCHA is the 8-order SCH model of geomagnetic anomalous field, the 5-order CGRF is the 5-order Taylor polynomial model of geomagnetic field.

\begin{tabular}{cccc}
\hline Model & $\sigma_{x}(\mathrm{nT})$ & $\sigma_{y}(\mathrm{nT})$ & $\sigma_{z}(\mathrm{nT})$ \\
\hline 8-order SCHN & 90.4 & 75.2 & 115.7 \\
8-order SCHA & 84.9 & 76.9 & 119.1 \\
5-order CGRF & 81.5 & 74.2 & 125.3 \\
\hline
\end{tabular}

of geomagnetic field) and the 8-order SCHA model (SCH model of geomagnetic anomalous field) are very closed for the same component.

Similarly, $K$ was respectively taken as $1 \sim 12$ for the Taylor polynomial model in model calculation. The results for various $K(1 \sim 12)$ were analyzed and compared, $K=5$ 
(a)

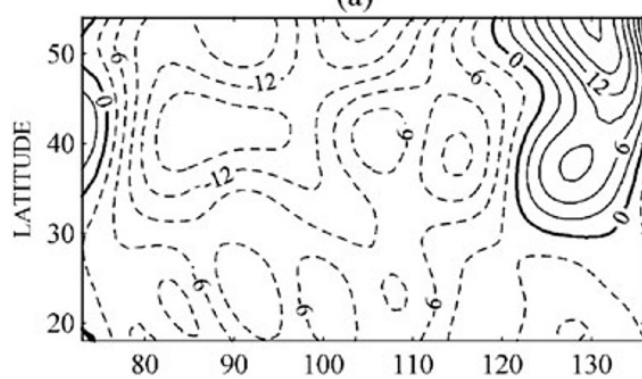

(c)

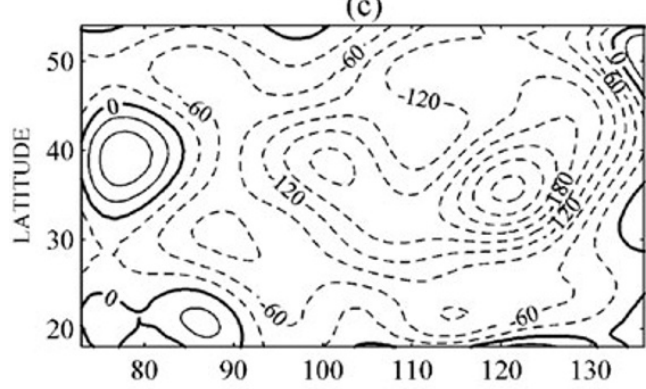

(e)

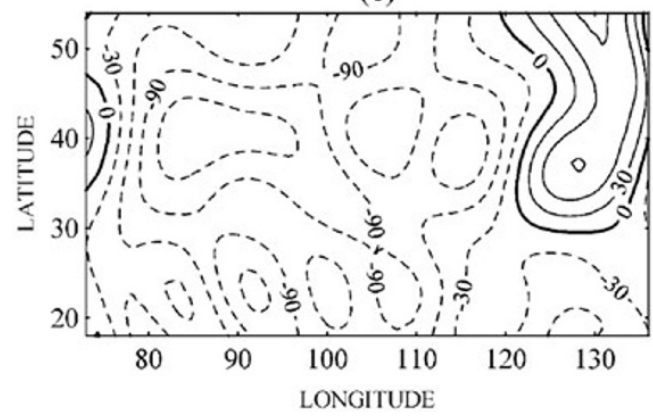

(b)

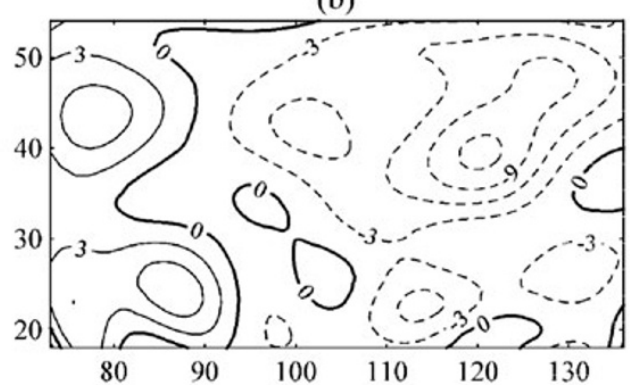

(d)

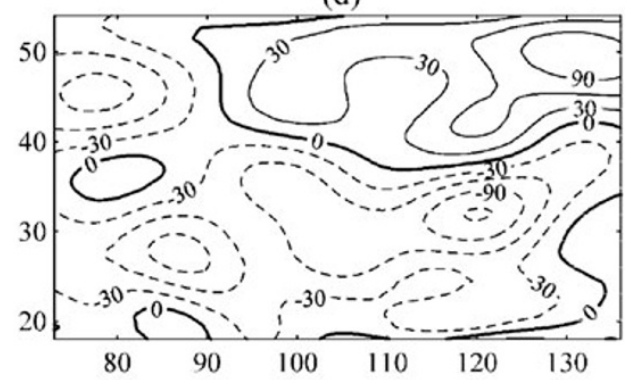

(f)

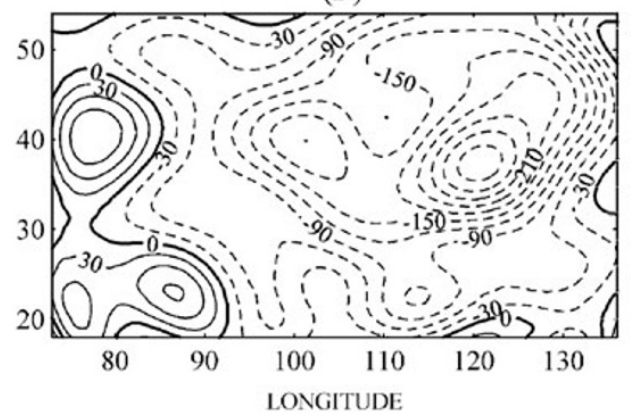

Fig. 5. Geomagnetic anomalous field derived from the 8-order SCHA model in China. (a) $\Delta D, \Delta=3^{\prime} ;$ (b) $\Delta I, \Delta=3^{\prime}$; (c) $\Delta F$, $\Delta=30 \mathrm{nT}$; (d) $\Delta X$, $\Delta=30 \mathrm{nT}$; (e) $\Delta Y, \Delta=30 \mathrm{nT}$; (f) $\Delta Z, \Delta=30 \mathrm{nT}$.

(a)

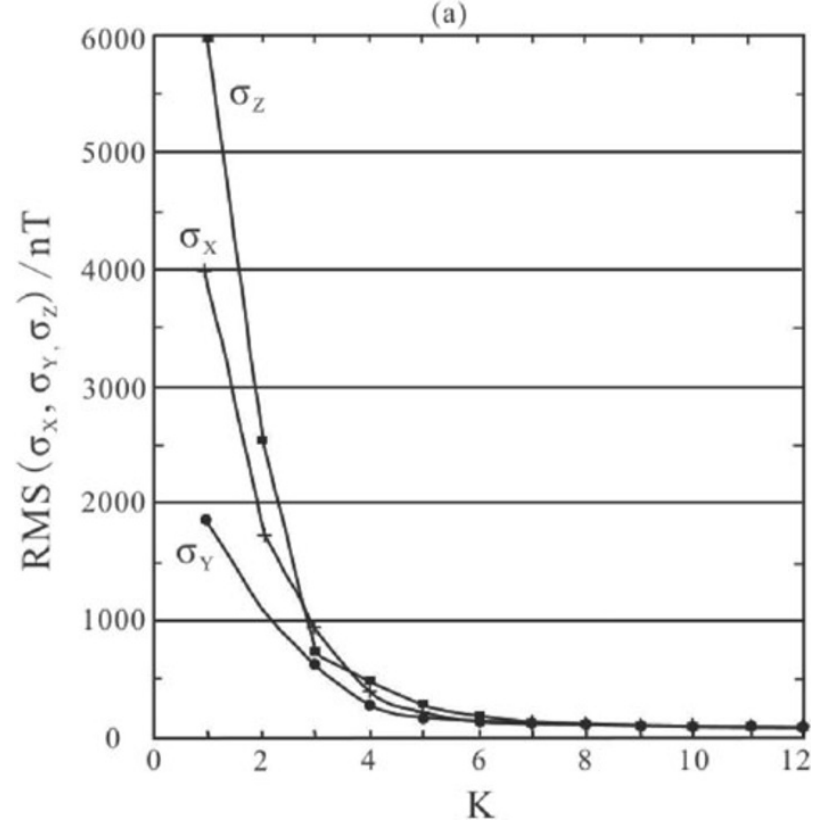

(b)

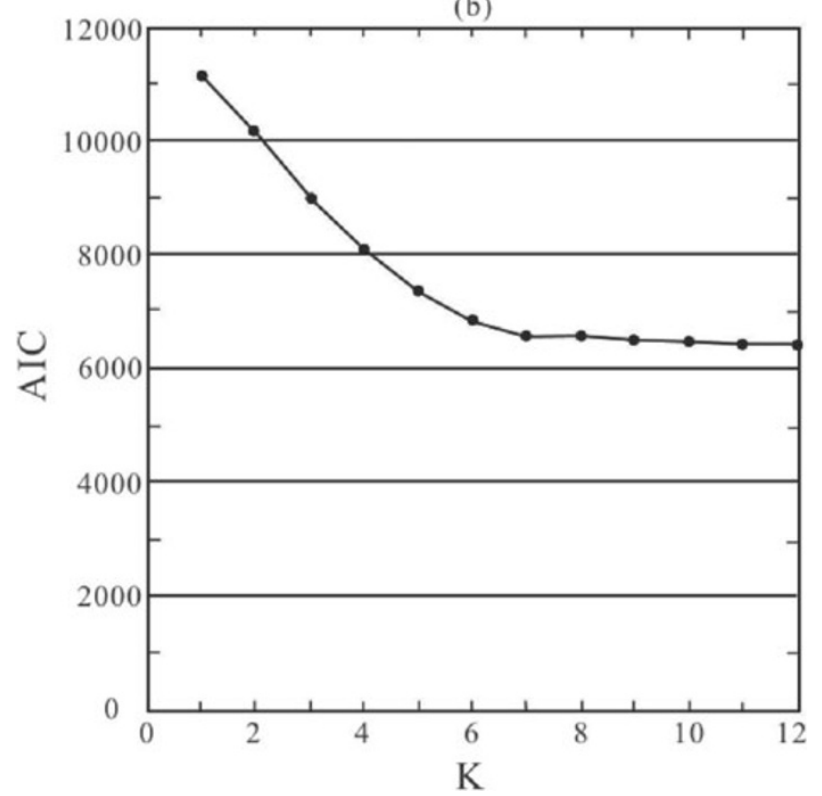

Fig. 6. (a) Decrease of $\sigma$ (RMS values) of the SCH model of the geomagnetic field with different truncation orders $K$. (b) Decrease of AIC of the SCH model of the geomagnetic field with different truncation order $K$. 
Table 6. AIC (Akaike Information Criteria) of spherical cap harmonic model for geomagnetic field in China.

\begin{tabular}{ccccccc}
\hline$K$ & 1 & 2 & 3 & 4 & 5 & 6 \\
$A I C$ & 11128.0 & 10135.0 & 8997.4 & 8120.2 & 7339.9 & 6839.6 \\
\hline$K$ & 7 & 8 & 9 & 10 & 11 & 12 \\
$A I C$ & 6554.0 & 6534.0 & 6511.4 & 6470.1 & 6426.9 & 6419.8 \\
\hline
\end{tabular}

(a)

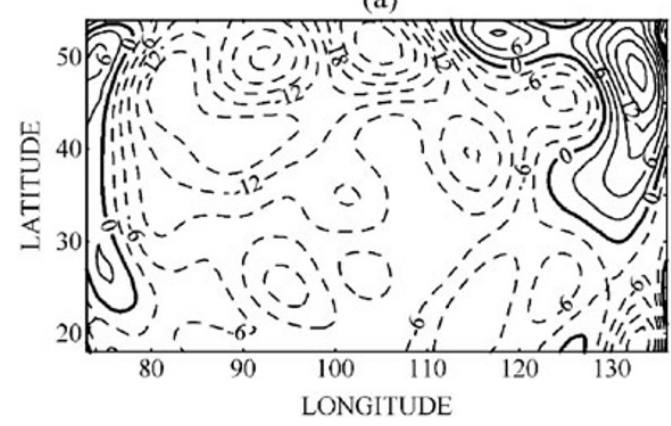

(b)

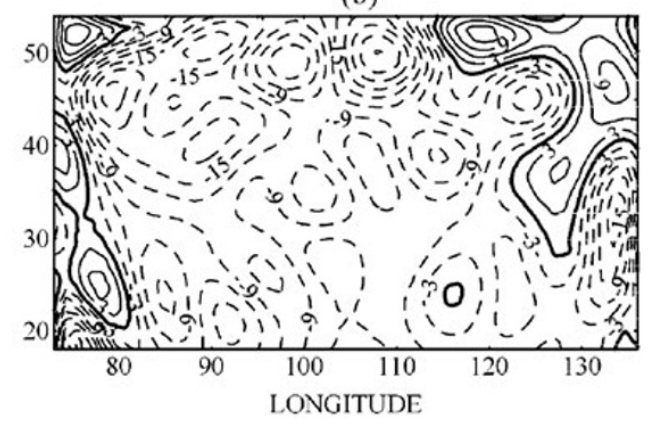

Fig. 7. Comparison of the declination distribution $\left(\Delta D, \Delta=3^{\prime}\right)$ of the $\mathrm{SCH}$ anomalous field models for (a) $K=9$; (b) $K=10$.

was finally determined for the Taylor polynomial model. In Table 7, the RMS values of the 5-order CGRF model (Taylor polynomial model of geomagnetic field) and the 8-order SCHN model (SCH model of geomagnetic field) are very closed for the same component.

\section{Conclusions}

The geomagnetic survey in China has obtained a lot of precise and reliable data by using the G-856 magnetometer, the DI magnetometer and GPS with good function and stability.

In establishing the geomagnetic field model, it is one of the key problems to determine the truncation order $K$. We comprehensively analyze and compare the mean square deviation between the model calculation value and the observation value, the AIC (Akaike Information Criterion) and the distribution of geomagnetic field for various $K$ taken as $1 \sim 12$. The results show that $K=8$ is optimum truncation order for the spherical cap harmonic model of geomagnetic field in China.

Taylor polynomial model of geomagnetic field is convenient in calculation and application. However, its deficiency is that it does not meet the potential theory of geomagnetic field and can only express the two-dimensional distribution of geomagnetic field. While the spherical cap harmonic model of geomagnetic field is complicated in calculation, but can meet the potential theory of geomagnetic field and express the three-dimensional structure of geomagnetic field. The 5-order Taylor polynomial model and the 8-order spherical cap harmonic model of geomagnetic field in China for 2003 are very similar. These two models have good consistency and complementation. Different users can choose one from the two models to meet their own demands.

Acknowledgments. This research was supported by the National Natural Science Foundation of China (40436016), the Ministry of Sciences and Technology of China and China Earthquake Admin- istration. We thank Prof. Hisashi Utada and Prof. Toshihiko Iyemori for their helps and good suggestions. We also appreciate the two referees, Dr. Yoichi Sasai and Dr. Toru Mogi for very valuable comments. Contribution No. 05FE3019 of Institute of Geophysics, China Earthquake Administration.

\section{References}

Akaike, H., A new look at the statiscal model identification, IEEE transactions on Automatic Control, 19(6), 716-723, 1974.

Alldredge, L., On regional magnetic charts, J. Geomag. Geoelectr., 39, 723-738, 1987.

An, Z., Review on geomagnetic survey, geomagnetic chart and model in China, Chinese J. Geophys., 44 (supplement), 45-50, 2001.

An, Z., Y. Xu, and Y. Wang, Derivation and analysis of the main geomagnetic field model in China for 1950-1980, Chinese J. Geophys. (ACTA Geophysica Sinica), 34, 585-593, 1991 (in Chinese).

Barton, C., Global and regional geomagnetic reference fields, Exploration Geophysics, 19, 401-416, 1988.

Chen, P., A detailed geomagnetic survey of Pehpei District, Szechuan, China, Chinese, J. Geophys. (Acta Geophysica Sinica), 1, 177-186, 1948.

Chen, Z., Review of geomagnetic surveys in China, Science Bulletin, 1, 99-120, 1944.

Chen, Z. and C. Liu, Preliminary report on the results of geomagnetic survey in China, 1946-1947, Chinese J. Geophys. (Acta Geophysica sinica), 1, 78-87, 1948.

Gao, J., Z. An, Z. Gu, W. Han, Z. Zhan, and T. Yao, Selection of geomagnetic normal field and calculation of geomagnetic anomalous field, Chinese J. Geophys., 48(1), 66-73, 2005.

Gu, Z., Z. An, J. Gao, W. Han, and Z. Zhan, Spherical cap harmonic analysis on the geomagnetic field in Beijing-Tianjin-Hebei region, Chinese J. Geophys., 47(6), 1128-1134, 2004.

Gu, Z., Z. Zhan, J. Gao, T. Yao, and W. Han, Application of GPS in geomagnetic survey, Earth Planets Space, 58, this issue, 721-727, 2006.

Haines, G., Regional magnetic field modeling: a review, J. Geomag. Geoelectr., 42, 1001-1018, 1990.

Haines, G. and L. Newitt, Canadian geomagnetic reference field 1985, J. Geomag. Geoelectr., 38(3), 895-921, 1986.

IAGA Division V, Working group 8, International Geomagnetic Reference Field 1995 revision, J. Geomag. Geoeletr., 47, 1257-1261, 1996.

IAGA Division V, Working Group 8, International Geomagnetic Reference Field 2000, Phys. Earth Planet. Inter., 120, 39-42, 2000.

IAGA Division V, Working Group 8, The $9^{\text {th }}$ Generation International Geomagnetic Reference Field, Geophys. J. Int., 155, 1051-1056, 2003.

Kote, M, and V. Haok, Modeling European magnetic repeat station and survey data by SCHA in search of time-variation anomalies, Phys. Earth 
Planet. Inter., 122(3-4), 205-220, 2000.

Langel, L., Main field, in Geomagnetism, edited by J. A. Jacobs, Vol. 1, pp. 249-512, Academic Press, London, 1987.

Miura, S., S. Ueki, T. Sato, K. Tachibana, and H. Hamaguchi, Crustal deformation associated with the 1998 seismo-volcanic crisis of Iwate Volcano, Northeastern Japan, as observed by a dense GPS network, Earth Planets Space, 52, 1003-1008, 2000.

Haines, G., Regional magnetic field modeling: a review, J. Geomag. Geoelectr., 42, 1001-1018, 1990.

Newitt, L. B., C. E. Barton, and J. Bitterly, in Guide for Magnetic Repeat Station Surveys, 112 pp., International Association of Geomagnetism and Aeronomy, 1996.

Tschu, K., On some advancement of Chinese geomagnetism and aeronomy during 1949-1979, Chinese J. Geophys. (Acta Gephysica sinica), 22,
326-335, 1979 (in Chinese).

Xia, G., S. Zheng, L. Wu, F. Zhang, and H. Wei, The geomagnetic field chart of China in 1980.0 and the mathematical model, Chinese J. Geophys. (Acta Geophysica Sinica), 31, 82-89, 1988 (in Chinese).

Xu, W., G. Xia, Z. An, G. Chen, F. Zhang, Y. Wang, Y. Tian, Z. Wei, S. $\mathrm{Ma}$, and H. Chen, Magnetic survey and China GRF2000, Earth Planets Space, 55, 215-217, 2003.

Z. Gu (e-mail: guzwg@sohu.com), Z. Zhan, J. Gao (e-mail: gaojt2000@yahoo.com.cn), W. Han (e-mail: whan@263.net), Z. An, T. Yao (e-mail: yaotq@sina.com), and B. Chen (e-mail: champion_chb@126.com) 